University of Wollongong

Research Online

Faculty of Engineering and Information

Faculty of Engineering and Information

Sciences - Papers: Part A

Sciences

$1-1-2014$

\title{
Short-term load forecasting using regression based moving windows with adjustable window-sizes
}

Dao Hoang $\mathrm{Vu}$

University of Wollongong, dhv972@uowmail.edu.au

Kashem M. Muttaqi

University of Wollongong, kashem@uow.edu.au

Ashish P. Agalgaonkar

University of Wollongong, ashish@uow.edu.au

Follow this and additional works at: https://ro.uow.edu.au/eispapers

Part of the Engineering Commons, and the Science and Technology Studies Commons

Research Online is the open access institutional repository for the University of Wollongong. For further information contact the UOW Library: research-pubs@uow.edu.au 


\title{
Short-term load forecasting using regression based moving windows with adjustable window-sizes
}

\begin{abstract}
This paper presents a regression based moving window model for solving the short-term electricity forecasting problem. Moving window approach is employed to trace the demand pattern based on the past history of load and weather data. Regression equation is then formed and least square method is used to determine the parameters of the model. In this paper, a new concept associated with cooling and heating degree is used to establish the relationship between electricity demand and temperature, which is one of the key climatic variables. In addition, Pearson's correlation has been employed to investigate the interdependency of electricity demand between different time periods. These analyses together with the data in the holiday period provide the supportive information for the appropriate selection of the window size. A case study has been reported in this paper by acquiring the relevant data for the state of New South Wales, Australia. The results are then compared with a neural network based model. The comparison shows that the proposed moving window approach with the different window sizes outperforms conventional neural network technique in small time scales i.e., from 30 minuntes to 1 day ahead.
\end{abstract}

\section{Keywords}

term, load, forecasting, regression, moving, short, windows, sizes, adjustable, window

\section{Disciplines \\ Engineering | Science and Technology Studies}

\section{Publication Details}

D. H. Vu, K. M. Muttaqi \& A. P. Agalgaonkar, "Short-term load forecasting using regression based moving windows with adjustable window-sizes," in Industry Applications Society Annual Meeting, 2014 IEEE, 2014, pp. 1-8. 


\title{
Short-term Load Forecasting using Regression based Moving Windows with Adjustable Window-Sizes
}

\author{
Dao H. Vu \\ Student Member, IEEE \\ dhv972@uowmail.edu.au \\ Kashem M. Muttaqi \\ Senior Member, IEEE \\ kashem@uow.edu.au \\ Ashish P. Agalgaonkar \\ Senior Member, IEEE \\ ashish@uow.edu.au \\ The authors are with Australian Power Quality and Reliability Center, School of Electrical Computer and \\ Telecommunications Engineering, University of Wollongong, NSW 2522, Australia
}

\begin{abstract}
This paper presents a regression based moving window model for solving the short-term electricity forecasting problem. Moving window approach is employed to trace the demand pattern based on the past history of load and weather data. Regression equation is then formed and least square method is used to determine the parameters of the model. In this paper, a new concept associated with cooling and heating degree is used to establish the relationship between electricity demand and temperature, which is one of the key climatic variables. In addition, Pearson's correlation has been employed to investigate the interdependency of electricity demand between different time periods. These analyses together with the data in the holiday period provide the supportive information for the appropriate selection of the window size. A case study has been reported in this paper by acquiring the relevant data for the state of New South Wales, Australia. The results are then compared with a neural network based model. The comparison shows that the proposed moving window approach with the different window sizes outperforms conventional neural network technique in small time scales i.e., from 30 minuntes to 1 day ahead.
\end{abstract}

Index Terms-- Climatic variables, Electricity demand forecasting, Moving window, Neural network

\section{INTRODUCTION}

Short-term electricity demand forecasting is crucial for power generation, operation and planning. The improvement in short-term demand forecasting accuracy leads to the reduction of operating costs [1], [2] and decrement in risk to the power system operations [3]. Furthermore, a short-term forecasting model is a critical decision support mean for operating electrical power system securely and efficiently. It also helps in coordinating the generation and area interchange to meet the load demand.

The short-term forecasting often deals with the time scale from few minutes up to few weeks ahead [4]. For this time scale, the load demand at a given hour is predominantly dependent on not only the load at previous hour, but also the load at the same hour on the previous day, and load at the same hour on the day with the same rank in the previous week [5]. In addition, the impacts of the exogenous variables, especially weather-related variables [6], and holidays events can cause enormous changes in load consumption. Consequently, accurate forecasting of load demand is more difficult due to the random variation of load and exogenous variables. As a result, consideration of all the impacts of these uncertainties on load forecasting, in short-term period, is necessary to develop the efficient model.

A considerable amount of literature on forecasting models and strategies has been reviewed in [7], and most of these methods can be applied to forecast the load in short-term period [4]. Regression-based method is employed in shortterm forecasting as it can represent the linear relationship between demand and temperature, one of the most important weather variables [8], [9]. This approach is based on the weather sensitive variables but it does not include the important information from the historical load data. On the other hand, the ARMA model [10] is widely used in shortterm forecasting since it can utilize the strong correlation between the load at a given time and load in previous times but it is non-weather sensitive model. While these methods exploit the linear relationship of load demand and other variables, neural network [11], [12] is employed in load forecasting because it has the ability to capture the non-linear relationship between demand and other variables. In regards to the development of neural network models, they can serve as forecasting benchmarks for short-term load forecasting [13], [14]. However, the neural network models are based on black box approach with minimal flexibility of use for network operators.

In this paper, a regression based moving window model is developed for solving the short-term forecasting problem. Moving window is used to correlate the demand at a given time to the demand at previous times and associated exogenous variables. The appropriate window sizes are selected based on demand pattern analysis and Pearson correlation analysis in conjunction with the holiday information. The balance point temperature is estimated based on the scatter plot between temperature and electricity demand data for every 30 minutes. The cooling and heating degree concepts are then used to linearize the relationship between temperature and demand. The Pearson correlation analysis has been employed to reveal the importance of climatic variables and historical demand data. The built window is then represented in the form of regression model which includes the significant variables. The forecasting results are then compared with the results obtained from neural network model to gauge the effectiveness of the proposed model.

The paper is organized as follows: Section II highlights 
the sensitivity of electricity demand to climatic variables. In section III, the electricity demand patterns and correlation are discussed. Section IV gives the description of the selected forecasting model. Section $\mathrm{V}$ provides the results and discussion, and Section VI details the concluding remarks.

\section{WEATHER VARIABLES}

The weather variables may have significant impacts on electricity demand, and temperature is one of the key variables since it controls atmospheric condition and affects other weather variables on the earth [15].

\section{A. Balance point Temperature}

Balance point temperature is a threshold temperature, at which the electricity demand is minimum. When the temperature increases or decreases from the balance point, the demand grows up due to cooling and heating requirements respectively. Ideally, a "V-shaped" trend curve [16] can be used to represent the balance point temperature. This balance point temperature is commonly considered to be around $18.3^{\circ} \mathrm{C}$ for the moderate environment, and at round $21.0^{\circ} \mathrm{C}$ for warmer environment [17]. In practical, the value of balance point temperature however, often varies in different regions due to geographical configuration. This value is also expected to be changed during a day due to the diversity in the dependence of electricity demand on temperature during each day. Therefore, consideration of these factors is necessary in estimating the balance point temperature. In the proposed study, balance point temperature has been estimated based on electricity demand and temperature data for typical time periods in one day.

\section{B. Cooling and Heating Degree}

According to the law of thermodynamics, if a substance weighing $m$ and having specific heat capacity of $C$ requires change in temperature from $t_{1}$ to $t_{2}$, then the requisite energy $E$ can be expressed as in (1)

$$
E=m^{*} C^{*}\left(t_{2}-t_{1}\right)
$$

With the same substance, $m$ and $C$ are constant, so the energy needed to change the temperature is proportional to the change of temperature. This implies that there is a strong relationship between the cooling and heating requirement with the change of temperature, and it leads to the use of cooling degree (CD) and heating degree (HD). Instead of using the specific unit of days [18] or hours [19], CD and HD can be used with respect to different units of time. In this paper, they are used to represent the relationship between demand and temperature every 30 minutes.

$\mathrm{CD}$ represents the requirement of cooling due to temperature being higher than the balance point temperature, and it is calculated as in (2).

$$
C D_{i}= \begin{cases}\left(T_{d i}-T_{b i}\right) & \text { if }\left(T_{d i}>T_{b i}\right) \\ 0 & \text { if }\left(T_{d i}<T_{b i}\right)\end{cases}
$$

Where, $T_{d i}$ is the average temperature at time $i$ of day $d, T_{b i}$ is the balance point temperature at time $i$.
Similarly, HD represents the heating energy requirement because of the temperature being lower than the balance point temperature, and it is calculated as in (3).

$$
H D_{i}= \begin{cases}\left(T_{b i}-T_{d i}\right) & \text { if }\left(T_{d i}<T_{b i}\right) \\ 0 & \text { if }\left(T_{d i}>T_{b i}\right)\end{cases}
$$

\section{ELECTRICITY DEMAND PATTERN}

Analysis the electricity demand pattern is critically important since it can reveal the seasonality of the demand, and provide more precious information for building the forecasting model. Specifically, including more seasonality into the forecasting model can significantly improve the accuracy of the forecasting results [13].

\section{A. Load Characteristics of Typical Days}

The load characteristic is normally different between working days and non-working days [20]. For the state of New South Wales, Australia, the differences in electricity demand patterns are shown in Fig. 1. It can be seen from this figure that there are two clear patterns of load demand which are working days (from Monday to Friday) and non-working days (Saturday, Sunday, and holiday). The demand requirement in the working days is greater than that in the non-working days. In the working days, the demand pattern is very strong, which can lead to nearly repetition of demand curve in the following day. In the non-working days, the demand on Sunday is generally lower than that on Saturday, but higher than that of a public holiday. Furthermore, the variation of load in one day is strongly affected by seasons such as Summer, Autumn, Winter and Spring.

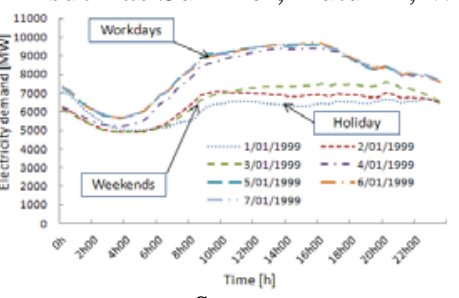

a. Summer

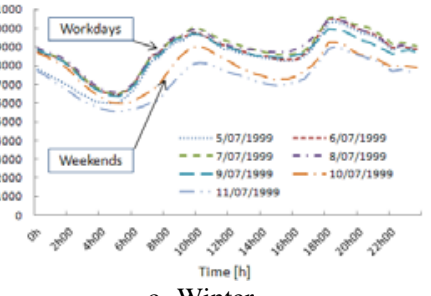

c. Winter

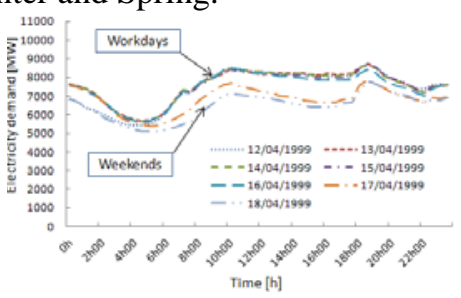

b. Autumn

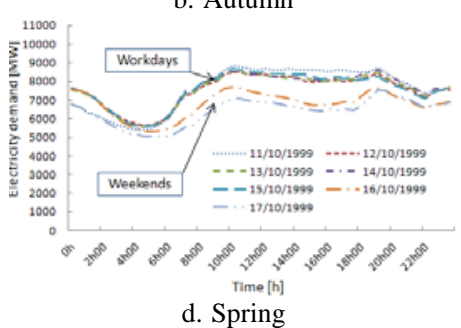

Fig. 1. The pattern of electricity demand in typical days in different seasons.

For more details in the winter season in Fig. 1.c, the demand gets peak at around 9:00am in the morning and around $18: 00 \mathrm{pm}$ in the afternoon, and the latter peak is higher than the former one. This is because of the habits using electric heater to warm up the office in the morning, and in the afternoon, not only warm up the house after arriving home, people often cook diner, and relax. Between the two peaks, the electricity consumption decrease due to 
more heat from the sunshine to the Earth surface, so less heating is required.

The variation of demand is considered in more detail for different days in one week in Fig. 2. This figure presents the average value, and the variation of demand for seven days in a week during four different seasons. It can be seen that the average demand in summer and winter is roughly higher than that in autumn and spring during a day. This difference is due to more heating required in winter, and more cooling needed in summer than the other seasons. It is also clear that the average demand is highest for winter due to extreme weather condition in this season in NSW, Australia. The demand variation in summer is only lower than that in winter in weekend, while during week days, it is quite similar for the both seasons. For the other seasons, autumn and spring, both the average value and variation are much lower than that in winter season, and they are similar to each other in each typical day.

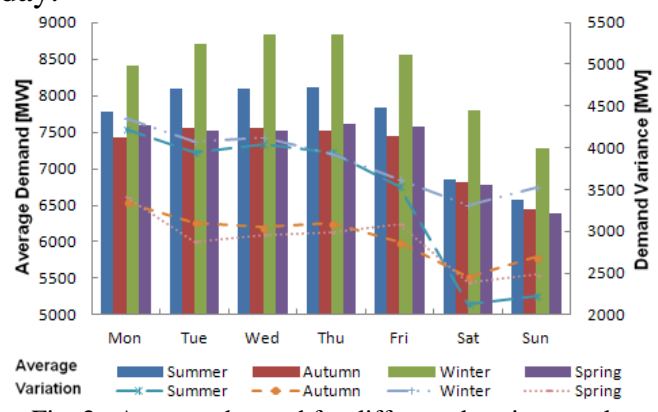

Fig. 2. Average demand for different days in a week.

\section{B. Correlation of Electricity Demand in Different Hours}

In order to estimate the interdependency among different variables, the Pearson correlation technique is commonly used. Using this technique, a correlation coefficient, which is between -1.0 and +1.0 , is generated to estimate the degree of correlation between different variables [21]. While +1.0 indicates a perfect positive correlation; -1.0 signifies a perfect inverse correlation; and 0 means no correlation. Commonly used formula of Pearson correlation is given in (4).

$$
r_{x y}=\frac{n \sum x y-\sum x \sum y}{\sqrt{\left[n \sum x^{2}-\left(\sum x\right)^{2}\right]\left[n \sum y^{2}-\left(\sum y\right)^{2}\right]}}
$$

where $r_{x y}$ is the correlation index between variables $x$ and $y$, and $n$ is the total number of data points.

The Pearson correlation between the historical data in different hours was calculated for January, 1999, and the results are given in Fig. 3. It can be seen from Fig. 3.a that the correlation of demand in different hours decrease vastly when the hour-gap between demand increases. This illustrates that the link between the demand at a given time and that at previous time is extremely loosen when the hourgap increases. Fig. 3.b which represents a zoom of correlation in a small time scale, shows that the correlation between the demands is very high (above 0.97 ) in a shortperiod of less than 2 hours. On the other words, the demand is much dependent on the very-close previous value of demand.

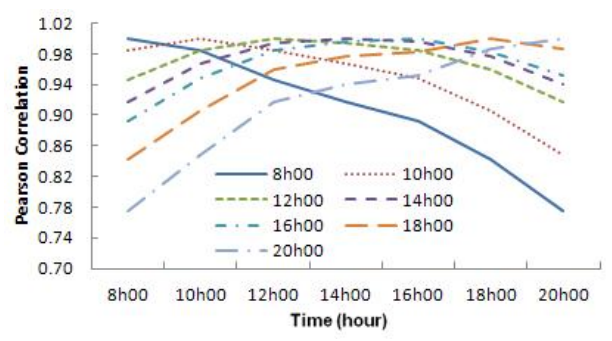

a. Large time scale

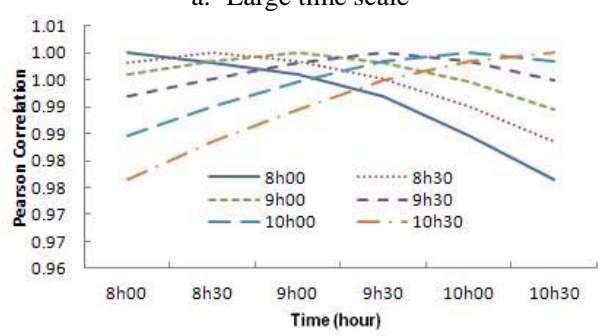

b. Small time scale

Fig. 3. Correlation of electricity demand at different time instants in January 1999.

\section{Correlation of Electricity Demand in Different Days}

Fig. 4 presents the correlation calculated for the typical working days to other days in January 1999. Although the correlation values are very low between these working days and non-working days (around 0.86), the correlation between the working days to other working days remains very high (above 0.95 in most of the time). This correlation value is even higher than that between hours in one day if the hourgap is big as shown in Fig. 3.a. This means that the demand value at the same hour in other working days is some time more significant than that at different hour in the same day with big hour-gap in term of correlation.

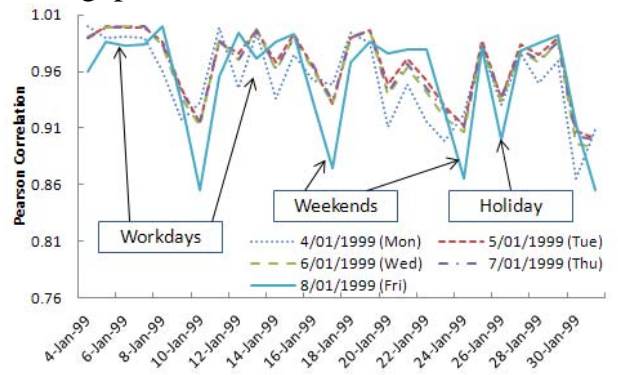

Fig. 4. Correlation of electricity demand in different days in January, 1999.

\section{SHORT-TERM LOAD FORECASTING}

In this section, the regression based moving window model is developed to be size-adjustable to forecast the electricity demand in short-term period. Moreover, the basic neural network model is then introduced to validate the developed model.

\section{A. Moving Windows Model}

As discussed in Section III, the demand has strong correlation with that in previous hours and previous days in prevalent patterns. This property of load can be utilized in moving window approach to advance the forecasting results. 
The size of the window indicates the link between the demands at a given time to the demand in the past. The typical window with the size of (3x3) is given in Fig. 5. In this example, the window is used to link the current demand to the demand at previous 30 minutes and three closest values of demands in two previous days. The smallest step of this window to move is 30 minutes.

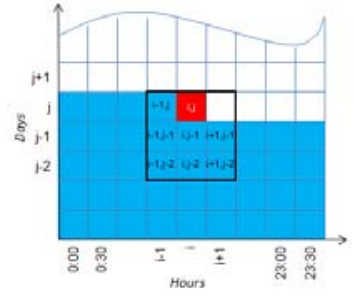

Fig. 5. Moving window approach.

From Fig.5, the representative equation for moving window approach can be described as in (5).

$$
\begin{aligned}
& y(i, j)=a_{0}+a_{1} y(i-1, j) \\
& +a_{2} y(i-1, j-1)+a_{3} y(i, j-1)+a_{4} y(i+1, j-1) \\
& +a_{5} y(i-1, j-2)+a_{6} y(i, j-2)+a_{7} y(i+1, j-2)+\varepsilon
\end{aligned}
$$

where $y(i, j)$ is demand value at time instant $i$ of day $j$, $a_{0}, a_{1}, a_{2}, \ldots a_{7}$ are the coefficients, and $\varepsilon$ is the error term.

In the other representation form, if we set:

$W_{1}=a_{1} y(i-1, j)$

$$
+a_{2} y(i-1, j-1)+a_{3} y(i, j-1)+a_{4} y(i+1, j-1)
$$$$
+a_{5} y(i-1, j-2)+a_{6} y(i, j-2)+a_{7} y(i+1, j-2)
$$

Then the equation (5) becomes:

$$
y(i, j)=a_{0}+W_{1}+\varepsilon
$$

For generalization, the moving window in Fig. 5 and (5) or (7) can be represented for the demand, temperature, or other variables.

In this study, the aim is to forecast the future demand, so the temperature and other variables can be used in conjunction with demand in a multiple-layers window. Fig. 6 shows the case of moving window with two layers which are demand and temperature. In this multiple-layers window, the temperature layer is used to support the demand layer. Other layers representing other variables can be attached in similar manner if necessary to provide more supportive information to electricity demand. Furthermore, not only the past data but also the updated forecasts of other variable can be selected based on the relationship with demand, and the size of the windows can be different in different layers.

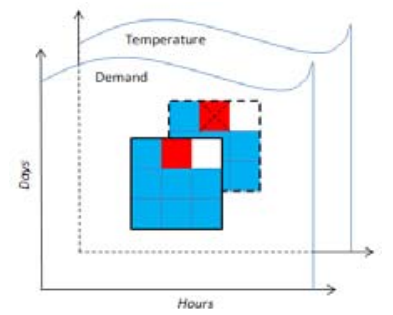

Fig. 6. Multiple layer moving window representation.

The representative equation for the multiple-layers window in Fig. 6 can be extended to $n$ layers, and the obtained equation is shown in (8).

$$
D(i, j)=a_{0}+W_{1}+W_{2}+\ldots+W_{n}+\varepsilon
$$

Where $D(i, j)$ is demand value at time instant $i$ of day $j$, $a_{0}$ is the constant coefficient, $W_{1}, W_{2}, \ldots, W_{n}$ are the representative of the window in different layers from 1 to $n$, and $\varepsilon$ is the error term.

Each of the value of windows $D(i, j)$ in (8) is representative for load demand at one specific time in a day. If each of them represents each hour of day, then 24 models can be used together to forecast 24 hours in one day [22]. In this article, with the fixed window size, the ability of the model only can be used to forecast one step ahead. It means that at one time instance, only the forecasting for next hour is available. Moreover, the article uses the temperature layer directly but not transform to HD and CD layers. This reduces the correlation of the associated variables to the demand, and lead to less accuracy results.

In this paper, the window is designed to be sizeadjustable, and it can extend the ability to forecast demand in a range from 30 minutes to 1 day ahead for each time instance of day. In addition, the size-adjustable moving window help improve the forecasting results significantly due to the appropriate tracing demand patterns. Furthermore, CD and HD layers are used to improve the utilization of dependence of temperature on demand. The process to choose the window size and to form the forecasting model is given in Fig. 7.

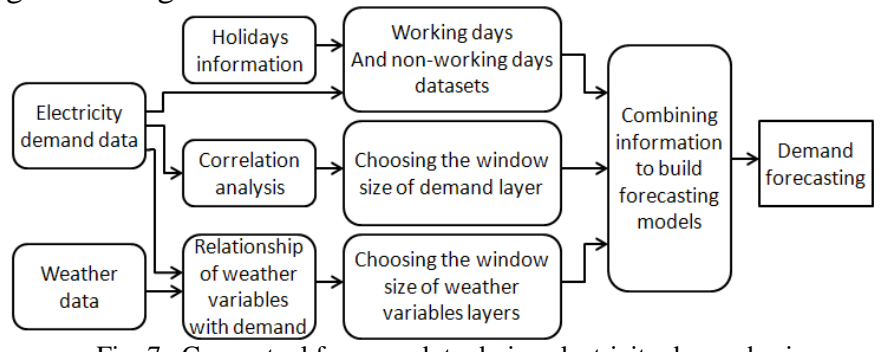

Fig. 7. Conceptual framework to derive electricity demand using multiple moving window approach.

\section{B. Neural Network Model}

In this paper, a neural network is introduced as a bench marking model to evaluate the accuracy of the above multiple moving window approach. The neural network is a feedforward neural network which composes of an input layer, one or more hidden layers, and an output layer [13]. As shown in Fig. 8, the $n$ inputs $x_{1}, x_{2}, \ldots x_{n}$ and a bias constant are fed to $m$ neurons $N_{1}, N_{2}, \ldots N_{m}$ in the hidden layer via a weight matrix $w_{j k}$. The output of the hidden layer is provided to the output layer which contains only one neuron $N_{o}$. The output layer produces the output $y$ which provides the information to forecast the electricity demand. In each neuron $N_{1}, N_{2}, \ldots N_{m}$ and $N_{o}$, there are two processes: (1) the inputs are summed by the summation function $S$, and (2) this summation is put through an activation function $f$ to produce 
the output.

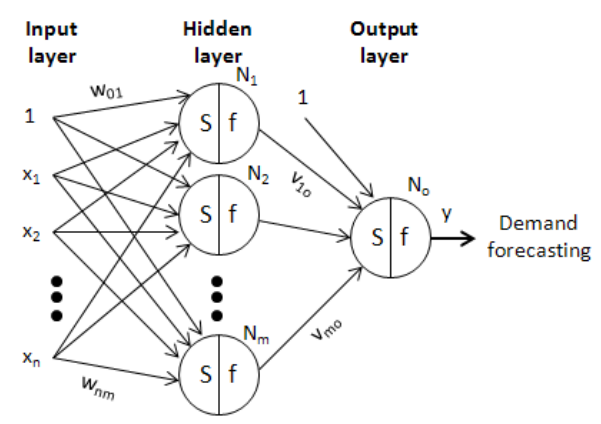

Fig. 8. Architecture of a neural network

\section{Mean Absolute Percentage Error (MAPE)}

MAPE vale is very useful in estimating the accuracy of the forecasting model. The formula of MAPE is given as in (9).

$$
\text { MAPE }=\frac{1}{n} \sum_{t=1}^{n}\left|\frac{A_{t}-M_{t}}{A_{t}}\right| * 100 \%
$$

Where $A_{t}$ and $M_{t}$ are actual demand and modeled demand at time $\mathrm{t}$ respectively, and $n$ is the number of forecasting points.

\section{RESULTS AND DISCUSSION}

A case study has been reported with the aid of historical data collected from the state of NSW, Australia for the years 1999 to 2000. The electricity demand data including all sectors, were collected from Australian energy market operator (AEMO) [23]. The climatic parameters at Sydney airport station [24] are assumed to be representing the entire state of NSW as around 75\% of population of NSW are in Sydney and the surrounding areas. Matlab and Microsoftexcel are employed to implement the requisite calculations. The data in year 1999 is used to train moving window and neural network models. These models are then tested on the data in year 2000. The results for two typical extreme seasons which are winter and summer, and entire period are presented.

\section{A. Balance Point Temperature}

The scatter plots were used to characterize the relationship between demand and temperature in different certain time interval, and the typical results are illustrated in Fig. 9. The different slopes in each graph reflect the different dependence during a day. From 0:00am to 4:00am, the demand seems to increase when the temperature decreases below a value of $20^{\circ} \mathrm{C}$, and it is nearly constant when the temperature is above this value. Around 6:00am, the dependence of demand on temperature is at minimum, and this can be considered as the zone which demand is independent from temperature as people often have deep sleep at this time. For the other hours of day (from 8:00am to 22:00pm), there seems to be strong relationship between demand and temperature in the V-shape due to differently elevated heating and cooling requirement for archiving the
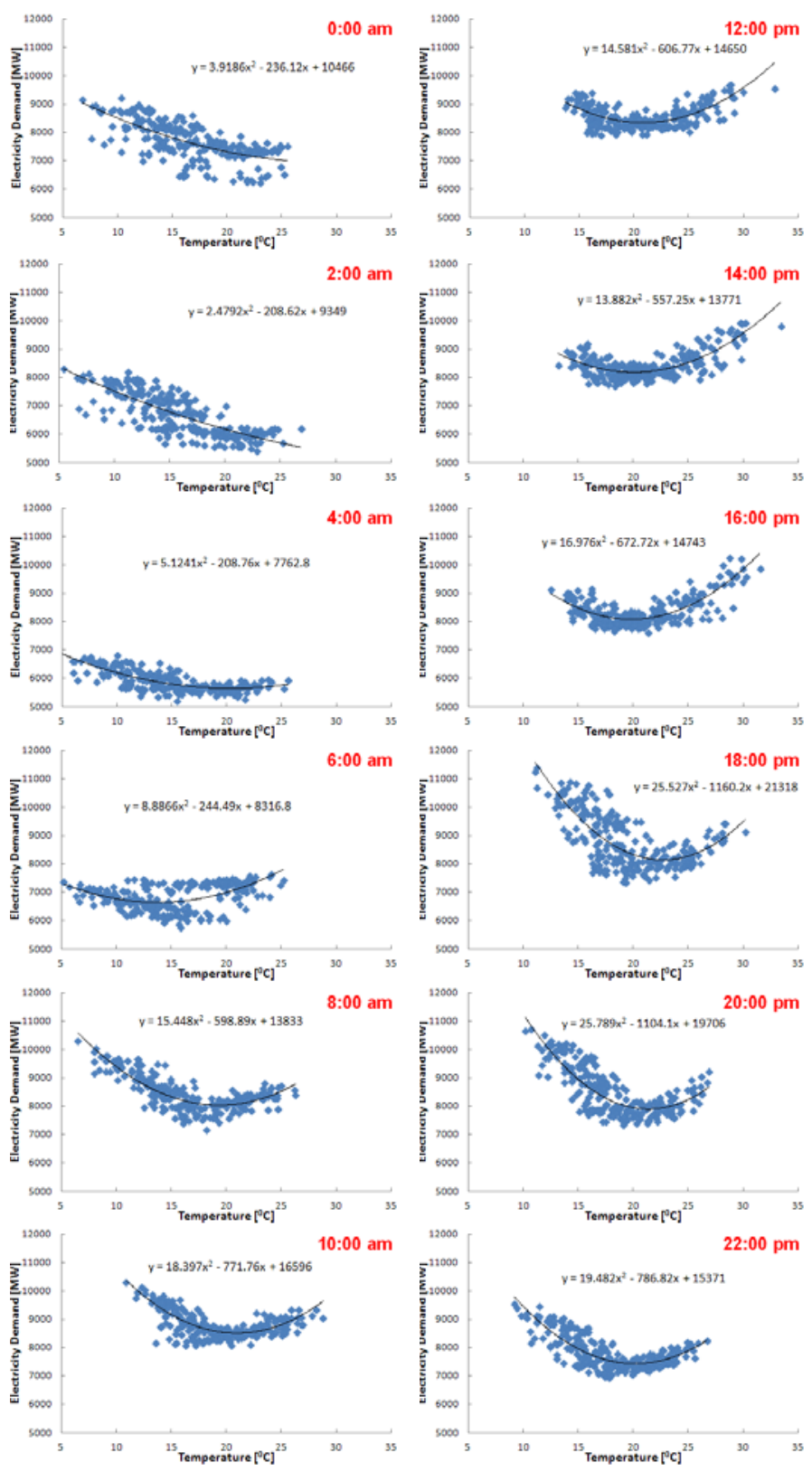

Fig. 9. Relationship between electricity demand and temperature at some typical time intervals.

living comfort. The balance point temperature is calculated for each of these hours, and the results are given in the Table I.

The balance point temperature varies from $19.2^{0} \mathrm{C}$ to $23.0^{\circ} \mathrm{C}$, and the average value of the balance point temperature is calculated to be $20.8^{\circ} \mathrm{C}$. This value can be used as balance point temperature for the other hours before 8:00am wherein almost zero cooling demand required. The balance point temperature is then used to transform the temperature to CD and HD in every 30 minutes, which are employed as inputs for the forecasting models.

Although the balance point temperature can be considered as the mean value $\left(20.8^{0} \mathrm{C}\right)$ for every hour, the dependence of 
demand on temperature is significantly different between different hours. It can be seen in Fig. 9 that the dependence is stronger in the hour of $18: 00 \mathrm{pm}$ and $20: 00 \mathrm{pm}$ due to steeper slope of the trend-line. This leads to different moving window models should be used to take into account of these differences in the dependence.

TABLE I

Balance Point Temperature In DifFerent Hours

\begin{tabular}{clc}
\hline \hline Hour & \multicolumn{1}{c}{ Equation } & $\begin{array}{c}\text { Balance point } \\
\text { temperature } \\
{\left[{ }^{0} \mathrm{C}\right]}\end{array}$ \\
\hline 8:00am & $\mathrm{y}=15.448 \mathrm{x} 2-598.89 \mathrm{x}+13833$ & 19.4 \\
9:00am & $\mathrm{y}=18.66 \mathrm{x} 2-717.48 \mathrm{x}+15233$ & 19.2 \\
10:00am & $\mathrm{y}=18.397 \mathrm{x} 2-771.76 \mathrm{x}+16596$ & 21.0 \\
11:00am & $\mathrm{y}=16.118 \mathrm{x} 2-674.42 \mathrm{x}+15479$ & 20.9 \\
$12: 00 \mathrm{pm}$ & $\mathrm{y}=14.581 \mathrm{x} 2-606.77 \mathrm{x}+14650$ & 20.8 \\
$13: 00 \mathrm{pm}$ & $\mathrm{y}=13.387 \mathrm{x} 2-544.55 \mathrm{x}+13802$ & 20.3 \\
$14: 00 \mathrm{pm}$ & $\mathrm{y}=13.882 \mathrm{x} 2-557.25 \mathrm{x}+13771$ & 20.1 \\
$15: 00 \mathrm{pm}$ & $\mathrm{y}=14.331 \mathrm{x} 2-563.13 \mathrm{x}+13620$ & 19.6 \\
$16: 00 \mathrm{pm}$ & $\mathrm{y}=16.976 \mathrm{x} 2-672.72 \mathrm{x}+14743$ & 19.8 \\
17:00pm & $\mathrm{y}=20.955 \mathrm{x} 2-877.2 \mathrm{x}+17306$ & 20.9 \\
$18: 00 \mathrm{pm}$ & $\mathrm{y}=25.527 \mathrm{x} 2-1160.2 \mathrm{x}+21318$ & 22.7 \\
19:00pm & $\mathrm{y}=23.993 \mathrm{x} 2-1102.9 \mathrm{x}+20685$ & 23.0 \\
20:00pm & $\mathrm{y}=25.789 \mathrm{x} 2-1104.1 \mathrm{x}+19706$ & 21.4 \\
21:00pm & $\mathrm{y}=23.017 \mathrm{x} 2-996.62 \mathrm{x}+18274$ & 21.6 \\
22:00pm & $\mathrm{y}=19.482 \mathrm{x} 2-786.82 \mathrm{x}+15371$ & 20.2 \\
23:00pm & $\mathrm{y}=11.705 \mathrm{x} 2-500.76 \mathrm{x}+12978$ & 21.4 \\
\hline \hline
\end{tabular}

B. Moving Window Approach

In this study, there will be four layers of moving window, including demand, $\mathrm{CD}, \mathrm{HD}$, and humidity layers. The improvement in performance of the model with participant of more layers is indicated in Fig. 10. It can be seen from this figure that the inclusion of all four layers is necessary to improve the accuracy of the results.

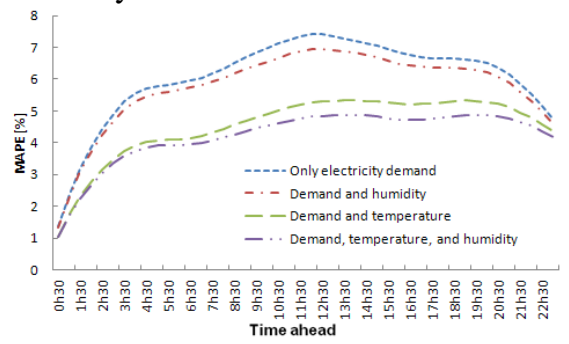

Fig. 10. Performance of model with the participant of different layers.

More practical works have been carried out with bigger size of the window for CD, HD, and humidity layer, but it is shown that those inclusions does not help improve the model further.

Keeping the size of the window in these layers for $1 \mathrm{x} 1$, and increase the size of window in demand layer, it can be found that the accuracy of the model was increased significantly for four first steps, and then does not increase any more with the bigger size of window. The limitation is at 5 refers back as in Fig. 11.

From Fig. 11, it is clear that the model has significant improvement when the number of past data points increase from 1 to 2, and small improvement from 2 to 4 . When the

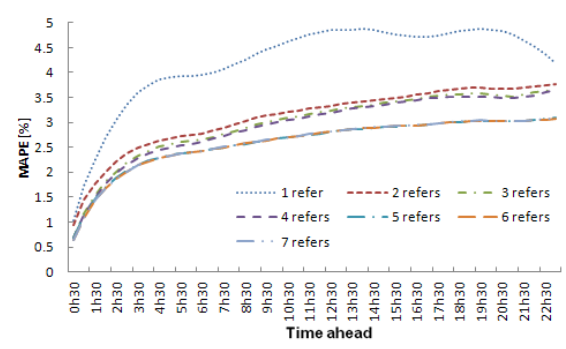

Fig. 11. Performance of model with different size of window in demand layer.

reference amends from 4 to 5 , an appreciably significant improvement of MAPE is noted. However, when the number is more than 5, there is not much improvement in MAPE. Accordingly, 5 data points, as in Table II, are used as optimal number for predicting demand using the proposed moving window approach.

TABLE II

CHOOSING THE WINDOW SIZE FOR SHORT-TERM FORECASTING Steps refer back

\begin{tabular}{cc}
\hline \hline & Steps refer back \\
\hline Demand window & $(i-h, j) ;(i-1, j-1) ;(i, j-1) ;(i, j-1) ;(i-1, j-2)$ \\
\hline CD window & $(i, j)$ \\
\hline HD window & $(i, j)$ \\
\hline Humidity window & $(i, j)$ \\
\hline \hline
\end{tabular}

Due to the different load pattern in different type of days as in Fig. 1 and 2, the window size is adjusted for capturing the demand in such types of days. For the week days from Tuesdays to Fridays, the window size can be selected as typical size in Table II. For the case of Mondays, if it is not the holiday, then the previous day (Sunday) is different type of day. If the moving window with the size as in Table II is applied for this situation, it will cause big error due to the different type of day having low correlation as shown in Fig. 4. In this case, the other working days in previous week (Friday for example) should be referred to. The same situation happens with Saturday and holidays. Those days should refer to the closet day in the same type of day to ensure the high correlation. For the holiday in this study, it is considered as same type with weekends. Therefore, there are two main types of days which are non-working days (including weekends and holidays), and working days (other days).

In addition to covering the type of days, the size of window can be adjusted to cover the different dependence of demand on the temperature as shown in Fig. 9 because in some time intervals, the demand seems to be independent from the temperature, so the window of CD and/or HD is not necessary.

\section{Neural Network Structure}

In order to compare with the moving window approach, the inputs of the network were chosen to be the same as the input of the moving window approach. The inputs for neural network using in this paper are CD, HD, humidity, hour of day, day of week, holidays, demand in the same time interval 
in previous week, the demand at the same hour in previous day.

The activation functions are chosen to be nonlinear sigmoid and linear function for the neurons in hidden layer and in output layer, respectively. The performance metric of the network is set to be mean absolute error (MAE). The back propagation algorithm was used to train the network which applies the Levenburg-Marquardt method as the optimization manner. The number of neurons in the hidden layer is chosen based on the performance of the network versus the number of the neurons as shown in Fig.12. This figure illustrates that with the increase of the number of neurons in hidden layer, the performance of the network is improved. Practical work leads to the optimum of the hidden network to be selected as 20 .

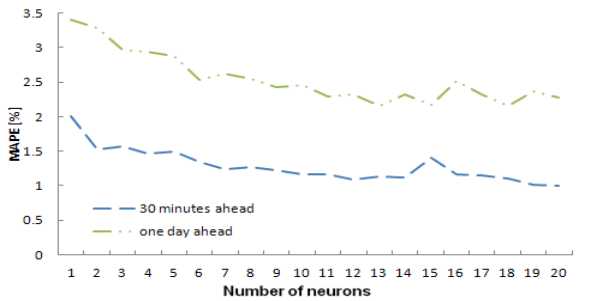

Fig. 12. Performance of neural network (MAPE) with different input in different time scales

\section{Half an Hour Ahead Forecasting}

Fig. 13 and 14 show the comparison of the modeled load using moving window and neural network methods respectively to actual demand in a typical summer week and typical winter week. It can be seen from these figures that the response of the moving window is very close to the actual demand. Even on middle day of Tuesday in Fig. 13, the neural network model overestimates the demand, but the result from moving window model still keep track with the actual demand.

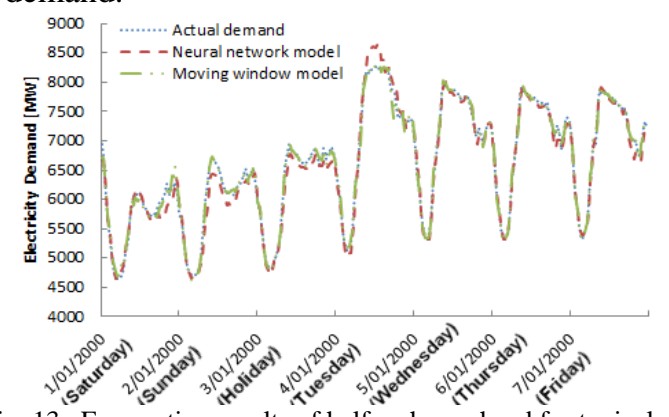

Fig. 13. Forecasting results of half an hour ahead for typical week in

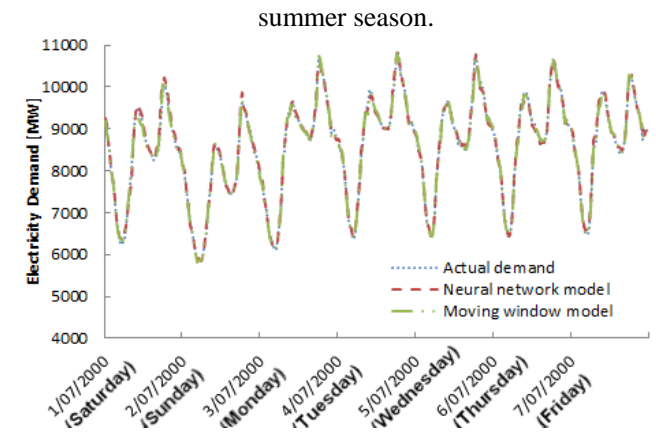

Fig. 14. Forecasting results of half an hour ahead for typical week in winter season.

The MAPE values are calculated for these typical periods of time and for whole period of year 2000 given in the Table III confirms the out-performance of moving window approach to the neural network model. This table shows that the accuracy of the moving window method is higher than that of neural network with smaller error values in each typical period.

The MAPE value of moving window approach is extremely small in comparison to that of neural network method, confirming the goodness, and robustness of this method in short-term demand forecasting one step of 30 minutes ahead. It also can be seen that for the both methods, the result in winter is better than that in summer season. This is due to the higher correlation of demand and temperature in winter than that in summer in NSW, Australia.

TABLE III

COMPARISON OF MAPE VALUES BETWEEN MOVING WINDOW AND NEURAL NETWORK METHODS FOR HALF AN HOUR AHEAD FORECASTING

\begin{tabular}{ccc}
\hline \hline & Neural network & Moving window \\
\hline Typical Winter week & 0.86 & 0.53 \\
\hline Typical Summer week & 1.50 & 0.79 \\
\hline Whole year 2000 & 1.04 & 0.59 \\
\hline \hline
\end{tabular}

\section{E. Twenty-four Hours Ahead Forecasting}

With the ability to adjust the size of the window, the moving window can give the forecasts for more than one step ahead. In this paper, the forecasting is done in a typical range from 30 minutes to 24 hours ahead. The comparison of moving window and neural network results can be viewed as in Fig.15.

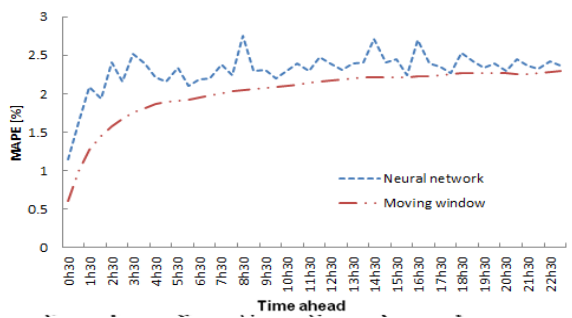

Fig. 15. Comparison of the performance of moving window and neural network results in a range of 30 minutes to 24 hours ahead

It can be seen from Fig. 15 that in the time scale from 30 minute to about 24 hours ahead, the moving window approach outperform the neural network. For a detail reference of forecasting value of 24 hours ahead, the results are presented in Fig. 16 and 17 for typical week of summer and winter, respectively. The MAPE values are given in the Table IV.

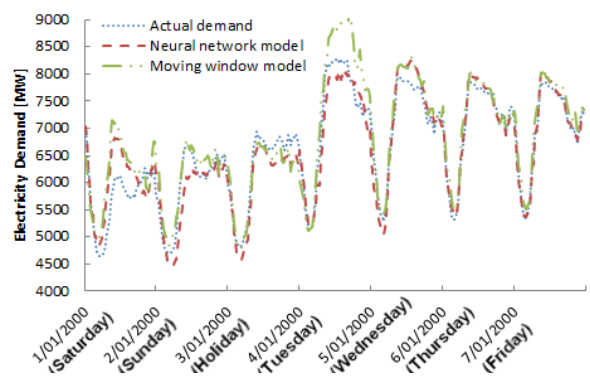


Fig. 16. Forecasting results of one day ahead for typical week in summer season.

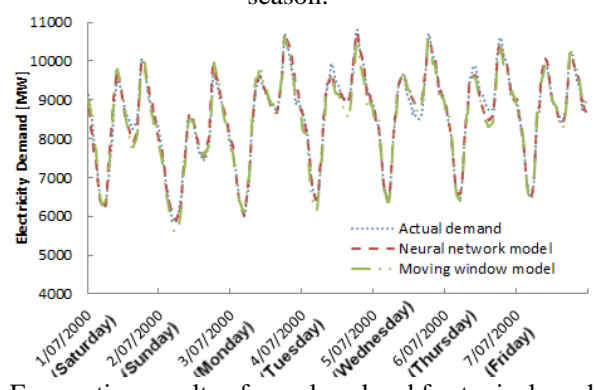

Fig. 17. Forecasting results of one day ahead for typical week in winter season.

TABLE IV

COMPARISON OF MAPE VALUES BETWEEN MOVING WINDOW AND NEURAL NETWORK METHODS FOR ONE DAYS AHEAD FORECASTING

\begin{tabular}{ccc}
\hline \hline & Neural network & Moving window \\
\hline Typical Winter week & 1.91 & 1.88 \\
\hline Typical Summer week & 3.50 & 4.26 \\
\hline Whole year 2000 & 2.31 & 2.30 \\
\hline \hline
\end{tabular}

The overall MAPE value for the proposed moving window model is small, especially for the small time scale i.e., 30 minutes ahead. This confirms the goodness of the model for short-term forecasting. For the longer time scale, the MAPE values obtained using proposed moving window model can increase faster and reach to more than $2 \%$ for one day ahead forecasting. At one day ahead forecasting, the result from the proposed method and the neural network method is quite similar as indicated in table IV.

\section{CONCLUSION}

This paper presents the regression based moving window approach to forecast the electricity demand in short-term period. The number of layers and the size of the window are selected based on the historical information and the correlation between demand and other related variables such as temperature. Also, the balance point temperature is calculated based on the equation representing the relationship between demand and temperature for a time interval of 30 minutes. The cooling and heating degree are introduced to linearize the relationship between demand and temperature. A case study for the state of NSW, Australia has been reported in the paper and the results show that the moving window is a promising approach in forecasting electricity demand for short-term period. The comparative studies conducted using the proposed model and the neural network model show the effectiveness of the proposed model in small time scale.

\section{ACKNOWLEDGMENT}

This work is supported by Hong Duc, Thanh Hoa - UOW research scholarship program.

\section{REFERENCES}

[1] B. F. Hobbs, S. Jitprapaikulsarn, S. Konda, V. Chankong, K. A. Loparo, and D. J. Maratukulam, "Analysis of the value for unit commitment of improved load forecasts," Power Systems, IEEE Transactions on, vol. 14, pp. 1342-1348, 1999.

[2] D. K. Ranaweera, G. G. Karady, and R. G. Farmer, "Economic impact analysis of load forecasting," Power Systems, IEEE Transactions on, vol. 12, pp. 1388-1392, 1997.

[3] A. P. Douglas, A. M. Breipohl, F. N. Lee, and R. Adapa, "Risk due to load forecast uncertainty in short term power system planning," Power Systems, IEEE Transactions on, vol. 13, pp. 1493-1499, 1998.

[4] M. A. Abu-El-Magd and N. K. Sinha, "Short-Term Load Demand Modeling and Forecasting: A Review," Systems, Man and Cybernetics, IEEE Transactions on, vol. 12, pp. 370-382, 1982.

[5] K. Srinivasan and R. Pronovost, "Short term load forecasting using multiple correlation models," Power Apparatus and Systems, IEEE Transactions on, vol. 94, pp. 1854-1858, 1975.

[6] R. Campo and P. Ruiz, "Adaptive Weather-Sensitive Short Term Load Forecast," Power Systems, IEEE Transactions on, vol. 2, pp. 592-598, 1987.

[7] L. Suganthi and A. A. Samuel, "Energy models for demand forecasting-A review," Renewable and Sustainable Energy Reviews, vol. 16, pp. 1223-1240, 2012.

[8] A. D. Papalexopoulos and T. C. Hesterberg, "A regression-based approach to short-term system load forecasting," in Power Industry Computer Application Conference, 1989. PICA '89, Conference Papers, 1989, pp. 414-423.

[9] M. O. Oliveira, D. P. Marzec, G. Bordin, A. S. Bretas, and D. Bernardon, "Climate change effect on very short-term electric load forecasting," in PowerTech, 2011 IEEE Trondheim, 2011, pp. 1-7.

[10] H. Shyh-Jier and S. Kuang-Rong, "Short-term load forecasting via ARMA model identification including non-Gaussian process considerations," Power Systems, IEEE Transactions on, vol. 18, pp. 673-679, 2003

[11] H. S. Hippert, C. E. Pedreira, and R. C. Souza, "Neural networks for short-term load forecasting: a review and evaluation," Power Systems, IEEE Transactions on, vol. 16, pp. 44-55, 2001

[12] J. W. Taylor and R. Buizza, "Neural network load forecasting with weather ensemble predictions," Power Systems, IEEE Transactions on, vol. 17, pp. 626-632, 2002.

[13] J. W. Taylor, "Triple seasonal methods for short-term electricity demand forecasting," European Journal of Operational Research, vol. 204, pp. 139-152, 2010.

[14] P.-F. Pai and W.-C. Hong, "Support vector machines with simulated annealing algorithms in electricity load forecasting," Energy Conversion and Management, vol. 46, pp. 2669-2688, 2005.

[15] L. Guan, J. Yang, and J. M. Bell, "Cross-correlations between weather variables in Australia," Building and Environment, vol. 42, pp. 10541070, 2007.

[16] T. Ahmed, K. M. Muttaqi, and A. P. Agalgaonkar, "Climate change impacts on electricity demand in the State of New South Wales, Australia," Applied Energy, vol. 98, pp. 376-383, 2012.

[17] D. J. Sailor, "Relating residential and commercial sector electricity loads to climate-evaluating state level sensitivities and vulnerabilities," Energy, vol. 26, pp. 645-657, 2001.

[18] H. Ching-Lai, S. J. Watson, and S. Majithia, "Analyzing the impact of weather variables on monthly electricity demand," Power Systems, IEEE Transactions on, vol. 20, pp. 2078-2085, 2005.

[19] S. J. Parkpoom and G. P. Harrison, "Analyzing the Impact of Climate Change on Future Electricity Demand in Thailand," Power Systems, IEEE Transactions on, vol. 23, pp. 1441-1448, 2008.

[20] F. M. Andersen, H. V. Larsen, and R. B. Gaardestrup, "Long term forecasting of hourly electricity consumption in local areas in Denmark," Applied Energy, vol. 110, pp. 147-162, 2013.

[21] P. M. Berthouex and L. C. Brown, Statistics for Environmental Engineers. Boca, Raton, London, New York, Washington, D.C.: Lewis Publishers, 2002.

[22] H. M. Al-Hamadi and S. A. Soliman, "Short-term electric load forecasting based on Kalman filtering algorithm with moving window weather and load model," Electric Power Systems Research, vol. 68, pp. 47-59, 2004.

[23] Australian Energy Market Operator. Available: http://www.aemo.com.au.

[24] Bureau of Meteology. Available: http://www.bom.gov.au. 